\title{
A Physics-Based Finite-State Abstraction for Traffic Congestion Control
}

\author{
Hossein Rastgoftar and Jean-Baptiste Jeannin
}

\begin{abstract}
This paper offers a finite-state abstraction of traffic coordination and congestion in a network of interconnected roads (NOIR). By applying mass conservation, we model traffic coordination as a Markov process. Model Predictive Control (MPC) is applied to control traffic congestion through the boundary of the traffic network. The optimal boundary inflow is assigned as the solution of a constrained quadratic programming problem. Additionally, the movement phases commanded by traffic signals are determined using receding horizon optimization. In simulation, we show how traffic congestion can be successfully controlled through optimizing boundary inflow and movement phases at traffic network junctions.
\end{abstract}

\section{INTRODUCTION}

Urban traffic congestion management is an active research area, and physics-based modeling of traffic coordination has been extensively studied by researchers over the past three decades. It is common to spatially discretize a network of interconnected roads (NOIR) using the Cell Transmission Model (CTM) which applies mass conservation to model traffic coordination [1], [2]. To control and analyze traffic congestion, the Fundamental Diagram is commonly used to assign a flowdensity relation at every traffic cell. While the Fundamental Diagram can successfully determine the traffic state for smallscale urban road networks, it may not properly function for congestion analysis and control in large traffic networks. Modeling of backward propagation, spill-back congestion, and shock-wave propagation is quite challenging. The objective of this paper is to deal with these traffic congestion modeling and control challenges. In particular, this paper contributes a novel integrative data-driven physics-inspired approach to $o b$ tain a microscopic data-driven traffic coordination model and resiliently control congestion in large-scale traffic networks.

Researchers have proposed light-based and physics-based control approaches to address traffic coordination challenges. Fixed-cycle control is the traditional approach for the operation of traffic signals at intersections. The traffic network study tool [3], [4] is a standard fixed-cycle control tool for optimization of the signal timing. Balaji and Srinivasan [5] and Chiu [6] offer fuzzy-based signal control approaches to optimize the green time interval at junctions. Physicsbased traffic coordination approaches commonly use the Fundamental Diagram to determine traffic state (flow-density relation) [7], [8], model dynamic traffic coordination [9], incorporate spillback congestion [10], [11], infuse backward propagation [12], [13] effects into traffic simulation, or specify

The authors are with the Department of Aerospace Engineering, Univer sity of Michigan, Ann Arbor, MI, 48109 USA e-mail: hosseinr@umich.edu. the feasibility conditions for traffic congestion control. Jafari and Savla [14] propose first order traffic dynamics inspired by mass flow conservation, dynamic traffic assignment [15], [16], and a cell transmission model [1], [17] to model and control freeway traffic coordination. Model predictive control (MPC) is an increasingly popular approach for model-based traffic coordination optimization [18]-[20]. Baskar et al. [21] apply MPC to determine the optimal platooning speed for automated highway systems (AHS). Furthermore, researchers have applied fuzzy logic [22]-[25], neural networks [26]-[29], Markov Decision Process (MDP) [30], [31], formal methods [32], [33] and mixed nonlinear programming (MNLP) [34] for model-based traffic management. Optimal control [14], [35] approaches have also been proposed. Rastgoftar et al. [36] model traffic coordination as a probabilistic process where traffic coordination is controlled only through boundary inlet nodes.

This paper studies the problem of traffic coordination and congestion control in a network of interconnected roads (NOIR). We model traffic coordination as a mass conservation problem governed by the continuity partial differential equation (PDE). Through spatial and temporal discretization of traffic coordination, this paper advances our previous work [36] by modeling traffic as a Markov process controlled through ramp meters (at boundary road elements) and traffic signals (at NOIR junctions). Given traffic feasibility conditions, MPC is applied to assign optimal boundary inflow such that traffic over-saturation is avoided at every NOIR road element. As the result, the optimal boundary inflow is continuously assigned as the solution of a constrained quadratic programming problem, and incorporated into traffic congestion planning. Given optimal boundary inflow, movement phase optimization is formulated as a receding horizon problem where discrete actions commanded by the traffic signals are assigned by minimization of coordination costs over a finite time horizon. Our proposed model ensures that traffic density is non-negative everywhere in the NOIR, if the traffic inflow is positive at every inlet boundary roads. Therefore, traffic coordination control can be commanded by a low computation cost.

This paper is organized as follows. Preliminary notions of graph theory presented in Section $[I$ are followed by traffic coordination modeling presented in Section III. Finite state abstraction of traffic coordination is presented in Section IV Ramp-based and signal-based traffic congestion control is presented in Section V. Simulation results are presented in Section VI followed by concluding remarks in Section VII 


\section{GRAPH THEORY NOTIONS}

Consider a NOIR with $m$ junctions defined by set $W=$ $\{1, \cdots, m\}$. An example of such a NOIR is shown in Fig. 1 (a). NOIR roads are identified by set $\mathcal{V}_{R}$ where $i \in \mathcal{V}_{R}$ is the index number of a road directed from an upstream junction to a downstream junction. Set $\mathcal{V}_{R}$ can be partitioned into a set of inlet boundary roads $\mathcal{V}_{i n}$ and a set of non-inlet roads $\mathcal{V}_{I}$ such that

$$
\mathcal{V}_{R}=\mathcal{V}_{i n} \bigcup \mathcal{V}_{I}
$$

We also define a single "Exit" road defined by singleton $\mathcal{V}_{E}$. Note that the "Exit" road does not represent a real road element (See Fig. 1 (a)); it is defined to model traffic coordination by a finite-state Markov process. We spatially discretize the NOIR using graph $\mathcal{G}(\mathcal{V}, \mathcal{E})$ with node set $\mathcal{V}=\mathcal{V}_{R} \cup \mathcal{V}_{E}$ and edge set $\mathcal{E} \subset \mathcal{V} \times \mathcal{V}$. Note that the nodes of graph $\mathcal{G}$ are the roads of our NOIR, and subsequently we use "road" and "node" interchangeably. Graph $\mathcal{G}$ is directed and the edge set $\mathcal{E}$ hold the following properties:

1) Traffic flow is directed from $\operatorname{road} i$, if $(i, j) \in \mathcal{E}$.

2) Real roads defined by set $\mathcal{V}_{R}$ are all unidirectional. Therefore, $(j, i) \notin \mathcal{E}$, if $(i, j) \in \mathcal{E}$.

Given graph $\mathcal{G}(\mathcal{V}, \mathcal{E})$, global in-neighbor, global outneighbor, inlet boundary nodes, non-inlet nodes, and "Exit" node are formally defined as follows:

Definition 1. Given edge set $\mathcal{E}$, the global in-neighbors of road $i$ are defined by set

$$
\mathcal{I}_{i}=\left\{j \in \mathcal{V}_{R}:(j, i) \in \mathcal{E}\right\} .
$$

Definition 2. Given edge set $\mathcal{E}$, the global out-neighbors of road $i$ are defined by set

$$
O_{i}=\{j \in \mathcal{V}:(i, j) \in \mathcal{E}\} .
$$

Definition 3. Inlet boundary roads have no in-neighbors at any time, and they are formally defined by set

$$
\mathcal{V}_{\text {in }}=\left\{i \in \mathcal{V}_{R}: \mathcal{I}_{i}=\emptyset \wedge O_{i} \neq \emptyset\right\} .
$$

Definition 4. Non-inlet roads have at least one in-neighbor and one out-neighbor at any time, and they are formally defined by set

$$
\mathcal{V}_{I}=\mathcal{V}_{R} \backslash \mathcal{V}_{\text {in }}
$$

Definition 5. The "Exit" node is formally defined as follows:

$$
\mathcal{V}_{E}=\left\{i \in \mathcal{V}: \mathcal{I}_{i} \neq \emptyset \wedge O_{i}=\emptyset\right\}
$$

where we assume that $\mathcal{V}_{E}$ is a singleton.

Without loss of generality, inlet boundary nodes are indexed from 1 through $N_{i n}$, non-inlet roads are indexed from $N_{i n}+1$ through $N$, and the "Exit" node is indexed by $N+1$. Therefore $\mathcal{V}_{\text {in }}=\left\{1, \cdots, N_{\text {in }}\right\}, \mathcal{V}_{I}=\left\{N_{\text {in }}+1, \cdots, N\right\}$, and $\mathcal{V}_{E}=\{N+1\}$ define the inlet, non-inlet, and "Exit" nodes, respectively. We use graph $\mathcal{G}(\mathcal{V}, \mathcal{E})$ to define interconnections between the NOIR roads, $\mathcal{V}=\mathcal{V}_{R} \cup \mathcal{V}_{E}$ and $\mathcal{E} \subset \mathcal{V} \times \mathcal{V}$ define nodes and edges of graph $\mathcal{G}$.

The NOIR shown in Fig. 1 contains 53 unidirectional "real" roads identified by set $\mathcal{V}_{R}=\{1, \cdots, 53\}$ and a virtual "Exit" node identified by set $\mathcal{V}_{E}=\{54\}$, i.e. $\mathcal{V}=\mathcal{V}_{R} \cup \mathcal{V}_{E}$. Note that roads $9, \cdots, 17 \in \mathcal{V}_{I} \subset \mathcal{V}_{R}$ are in-neighbors to the "Exit" node $54 \in \mathcal{V}_{E}$, as represented by the dotted lines. Thus

$$
\mathcal{I}_{54}=\{9, \cdots, 17\} .
$$

Inlet nodes are identified by $\mathcal{V}_{i n}=\{1, \cdots, 8\}$ and $\mathcal{V}_{I}=$ $\{9, \cdots, 53\}$ defines all non-inlet roads.

Movement Phase Rotation: At each intersection, we define movement phases representing the different possible configurations of traffic light states at that intersection or, equivalently, the different possible paths that are allowed at that intersection. For instance, in the example of Fig. 11, intersection number 10 has three lights - at the ends of roads 33, 35 and 50 - and three different movement phases:

- the first movement phase $\lambda_{10,1}$ corresponds to a green light at the end of road 50, and red lights at the ends of roads 33 and 35; equivalently, cars are allowed to circulate from road 50 to roads 34,13 or 36 , and no other circulation is allowed;

- the second movement phase $\lambda_{10,2}$ corresponds to a green light at the end of road 35, and red lights at the end of roads 33 and 50; cars are only allowed to circulate from road 35 to either road 13 or 36 ;

- the third movement phase $\lambda_{10,3}$ corresponds to a green light at the end of road 33, and red lights at the end of roads 35 and 50 to be red; cars are only allowed to circulate from road 33 to either road 13 or 34 .

Those three movement phases define the three possible configurations of the lights at intersection number 10, and over time the lights of intersection 10 alternatively go over those movement phases.

Formally, let $\mathcal{M}_{i n, j} \subset \mathcal{V}_{R}$ define incoming roads and $\mathcal{M}_{\text {out }, j} \subset \mathcal{V}_{R}$ define outcoming roads at junction $j \in \mathcal{W}$. Every junction $j$ is associated with $\mu_{j}$ movement phases that can be commended by the traffic signals. The set of edges $\lambda_{j, k} \subset \mathcal{M}_{i n, j} \times \mathcal{M}_{\text {out }, j} \subset \mathcal{E}$ is the $k$-th movement phase commanded at junction $j \in \mathcal{W}$ where $k=1, \cdots, \mu_{j}$. Movement phases at junction $j \in \mathcal{W}$ are defined by finite set $\Lambda_{j}$ as follows:

$$
\boldsymbol{\Lambda}_{j}=\bigcup_{k=1}^{\mu_{j}}\left\{\lambda_{j, k}\right\}=\left\{\lambda_{j, 1}, \ldots, \lambda_{j, \mu_{j}}\right\}
$$

where $j \in \mathcal{W}$ and $k=1, \cdots, \mu_{j}$. Note that $\boldsymbol{\Lambda}_{j}$ is a set of subsets of edge set $\mathcal{E}$, i.e., is contained in the powerset of $\mathcal{E}$. We can define

$$
\boldsymbol{\Lambda}=\boldsymbol{\Lambda}_{1} \times \cdots \times \boldsymbol{\Lambda}_{m}
$$

as the set of all possible movement phases across the NOIR. Transitions of movement phases are cyclic at every junction $j \in \mathcal{W}$, and defined by cycle graph $C_{j}\left(\boldsymbol{\Lambda}_{j}, \boldsymbol{\Xi}_{j}\right)$ with node set $\boldsymbol{\Lambda}_{j}$ and edge set

$$
\boldsymbol{\Xi}_{j}=\left\{\left(\lambda_{j, 1}, \lambda_{j, 2}\right), \cdots,\left(\lambda_{j, \mu_{j}-1}, \lambda_{j, \mu_{j}}\right),\left(\lambda_{j, \mu_{j}}, \lambda_{j, 1}\right)\right\}
$$

Intuitively, first $\lambda_{j, 1}$ is the active movement phase defining the current traffic light states and equivalent authorized paths at junction $j \in \mathcal{W}$; then the active movement phase is switched 


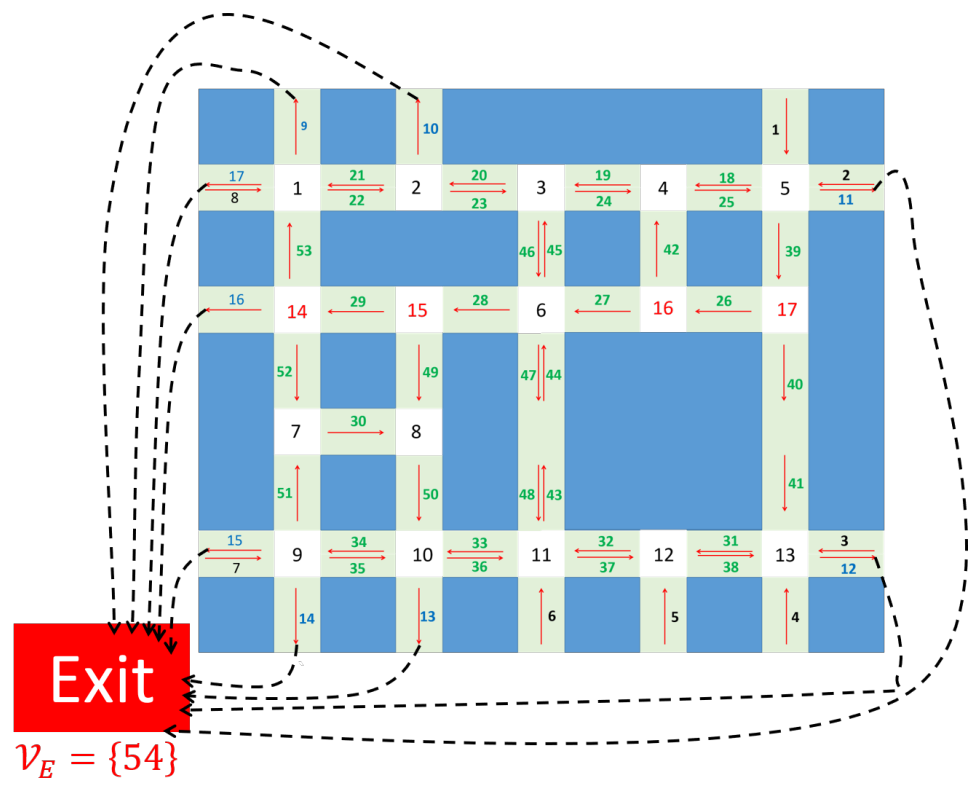

(a)

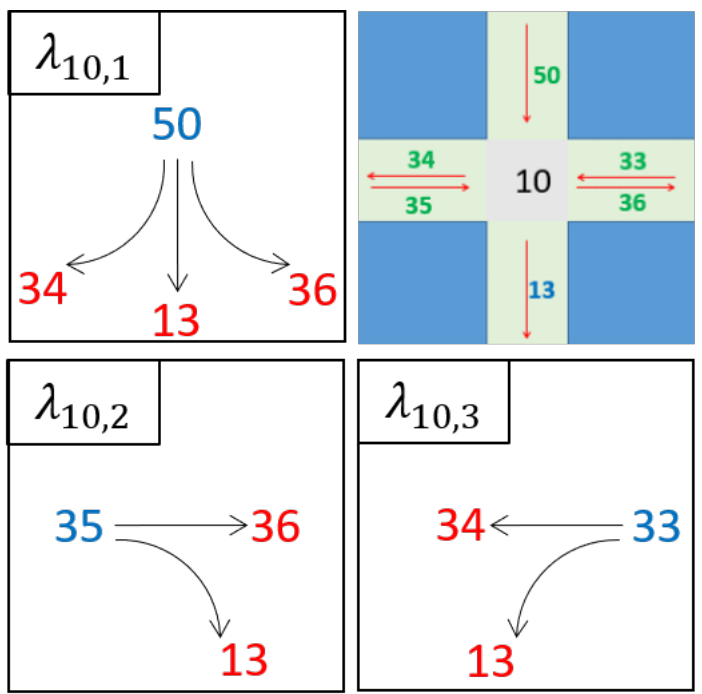

(b)

Fig. 1: (a) Example NOIR with 53 unidirectional roads. (b) Three possible movement phases at junction $10 \in \mathcal{W}$.

to $\lambda_{j, 2}$, then to $\lambda_{j, 3}, \ldots$, then to $\lambda_{j, \mu_{j}}$, then back to $\lambda_{j, 1}$ to restart the cycle.

Fig. 1] (b) shows all possible movement phases at junction $10 \in \mathcal{W}$ of the NOIR shown in Fig. 1 (a), where $\mathcal{W}=\{1, \cdots, 13\}$ defines the junctions. The incoming and outcoming roads are defined by set $\mathcal{M}_{i n, 10}=\{33,35,50\}$ and $\mathcal{M}_{\text {out }, 10}=\{13,34,36\}$, respectively. There are three movement phases $\lambda_{10,1}=\{(50,34),(50,13),(50,36)\} \subset \mathcal{E}, \lambda_{10,2}=$ $\{(35,13),(35,36)\} \subset \mathcal{E}$, and $\lambda_{10,1}=\{(33,13),(33,34)\} \subset \mathcal{E}$. Note that U-turns are disallowed at every junction of the Example NOIR shown in Fig. 1

Movement Phase Activation Time: It is assumed that movement phase $\lambda_{j, k} \in \boldsymbol{\Lambda}_{j}\left(k=1, \cdots, \mu_{j}\right)$ cannot be active more that $T_{L, j}$ time steps, where $T_{L, j} \in \mathbb{N}$ is equivalent to $T_{L, j} \Delta T$ seconds, and $\Delta T$ is a known constant time step interval. Because movement rotation is cyclic at every junction $j \in \mathcal{W}$, we define the maximum activation time $T_{L, j}$ for every movement phase at NOIR junction $j \in \mathcal{W}$. Define $T_{j}$ as the activation time of a movement phase at junction $j \in \mathcal{W}$, where $T_{j} \leq T_{L, j}$. Note that $T_{j}$ is independent of index $k \in\left\{1, \cdots, \mu_{j}\right\}$ and is counted from the start time of a movement phase $\lambda_{j, k}$ at junction $j \in \mathcal{W}$. Given $T_{j}$ and $T_{L, j}$, we define activation index

$$
j \in \mathcal{W}, \quad \tau_{j}=\left\lfloor\frac{T_{j}}{T_{L, j}}\right\rfloor \in\{0,1\}
$$

at every intersection $j \in \mathcal{W}$, where $\lfloor\cdot\rfloor$ denotes the floor function. Because $T_{j} \leq T_{L, j}, \tau_{j} \in\{0,1\}$ is a binary variable assigning whether the active movement phase must be overridden or not. If $\tau_{j}=0$, the current movement $\lambda_{j, k}\left(k=1, \cdots, \mu_{j}, j \in \mathcal{W}\right)$ can still remain active. Otherwise, the active movement phase is overridden and the next movement phase must be selected.
The network movement phase is denoted by $\lambda=$ $\left(\lambda_{1}, \cdots, \lambda_{m}\right) \in \boldsymbol{\Lambda}$ where $\lambda_{j} \in \boldsymbol{\Lambda}_{j}$ and $j \in \mathcal{W}$. We define the switching communication graph $\mathcal{G}_{\lambda}\left(\mathcal{V}, \mathcal{E}_{\lambda}\right)$ to specify the inter-road connection under movement phase $\lambda \in \boldsymbol{\Lambda}$, where $\mathcal{E}_{\lambda} \subset \mathcal{E}$ defines the edges of graph $\mathcal{G}$. Per movement phase definition given in (7), $\mathcal{E}_{\lambda}=\cup_{k=1}^{m} \lambda_{k}$. In-neighbors and outneighbors of road (or Exit node) $i \in \mathcal{V}$ is defined by the following sets:

$$
\begin{array}{ll}
i \in \mathcal{V}, \lambda \in \Lambda, & \mathcal{I}_{i, \lambda}=\left\{j \in \mathcal{V}_{R}:(j, i) \in \mathcal{E}_{\lambda}\right\}, \\
i \in \mathcal{V}, \lambda \in \Lambda, & O_{i, \lambda}=\left\{j \in \mathcal{V}:(i, j) \in \mathcal{E}_{\lambda}\right\} .
\end{array}
$$

Given the above definitions, for any $\lambda \in \Lambda, I_{i, \lambda} \subset I_{i}$ and $O_{i, \lambda} \subset$ $O_{i}$, thus:

1) for every $\lambda \in \Lambda$, in-neighbor set $\mathcal{I}_{i, \lambda}=\emptyset$ if $i \in \mathcal{V}_{i n}$;

2) for every $\lambda \in \Lambda$, out-neighbor set $O_{i, \lambda}=\emptyset$ if $i \in \mathcal{V}_{E}$.

\section{III.TRAFFIC COORDINATION MODEL}

We use the mass conservation law to model traffic at every NOIR road element $i \in \mathcal{V}$. Let $\rho_{i}, y_{i}$, and $z_{i}$ denote traffic density, traffic inflow, and traffic outflow at every road element $i \in \mathcal{V}$. Traffic dynamics governed by mass conservation is:

$$
\rho_{i}(k+1)=\rho_{i}(k)+y_{i}(k)-z_{i}(k),
$$

where

$$
\begin{aligned}
& z_{i}(k)= \begin{cases}p_{i}(\lambda) \rho_{i}(k) & i \in \mathcal{V}_{R}, \forall \lambda \in \boldsymbol{\Lambda} \\
\rho_{i}(k)+y_{i}(k) & i \in \mathcal{V}_{E}, \forall \lambda \in \boldsymbol{\Lambda}\end{cases} \\
& y_{i}(k)= \begin{cases}u_{i}(k) & i \in \mathcal{V}_{i n}, \forall \lambda \in \boldsymbol{\Lambda} \\
\sum_{j \in I_{i, \lambda}} q_{i, j}(\lambda) z_{j}(k)+d_{i} & i \in \mathcal{V} \backslash \mathcal{V}_{i n}, \forall \lambda \in \boldsymbol{\Lambda}\end{cases}
\end{aligned}
$$


and inflow $y_{i} \geq 0$ at road element $i \in \mathcal{V}_{i n}$ has the following properties:

1) If $i \in \mathcal{V}_{i n}, y_{i}=u_{i}$ can be controlled by a ramp meter.

2) If $i \in \mathcal{V}_{I}, d_{i} \geq 0$ is given as a non-zero-mean Gaussian process.

Note that $d_{i}$ is uncontrolled at road element $i \in \mathcal{V}_{R} \backslash \mathcal{V}_{\text {in }}$. Variable $p_{i}(\lambda) \in[0,1]$ is the traffic outflow probability, and $q_{i, j}(\lambda)$ is the outflow fraction of road element $j$ directed towards $i \in O_{j, \lambda}$ when $\lambda \in \boldsymbol{\Lambda}$ is the active movement phase over time interval $\left[t_{k}, t_{k+1}\right]$. Note that

$$
\sum_{j \in O_{i, \lambda}} q_{j, i}(\lambda)=1
$$

for every $\lambda \in \boldsymbol{\Lambda}$. We define $\mathbf{P}(\lambda)=$ $\operatorname{diag}\left(p_{1}(\lambda), \cdots, p_{N}(\lambda), p_{N+1}(\lambda)\right)$, where $p_{N+1}(\lambda)=0$ $\forall \lambda \in \Lambda$. This implies that the outflow of the exit node is zero. Also, matrix $\mathbf{Q}(\lambda)=\left[q_{i, j}(\lambda)\right] \in \mathbb{R}^{(N+1) \times(N+1)}$ is non-negative, and

$$
q_{N+1, j}(\lambda)=\left\{\begin{array}{ll}
1 & j=N+1 \in \mathcal{V}_{E} \\
0 & \text { otherwise }
\end{array} .\right.
$$

Eq. (14) implies that traffic does not flow from the exit node $N+1 \in \mathcal{V}_{E}$ to any other element $j \in \mathcal{V}_{R} \backslash \mathcal{V}_{E}$. The traffic network dynamics is given by

$$
\mathbf{x}(k+1)=\mathbf{A}(\lambda) \mathbf{x}(k)+\mathbf{g}(k)
$$

where $\quad \mathbf{x}(k)=\left[\begin{array}{lll}\rho_{1}(k) & \cdots & \rho_{N+1}(k)\end{array}\right]^{T} \quad$ and $\mathbf{g}=\left[\begin{array}{ll}\mathbf{g}_{R}^{T} & g_{N+1}\end{array}\right]^{T}=\left[g_{i}\right] \in \mathbb{R}^{(N+1) \times 1}$ is defined as follows:

$$
g_{i}(k)= \begin{cases}u_{i}(k) & i \in \mathcal{V}_{i n} \\ d_{i}(k) & i \in \mathcal{V}_{R} \backslash \mathcal{V}_{i n} . \\ 0 & i \in \mathcal{V}_{E}\end{cases}
$$

Also,

$$
\mathbf{A}(\lambda)=\mathbf{I}-\mathbf{P}(\lambda)+\mathbf{Q}(\lambda) \mathbf{P}(\lambda)=\left[\begin{array}{ll}
\mathbf{C}(\lambda) & \mathbf{0} \\
\mathbf{D}(\lambda) & 1
\end{array}\right],
$$

where every column of non-negative matrix $\mathbf{A}: \boldsymbol{\Lambda} \rightarrow$ $\mathbb{R}^{(N+1) \times(N+1)}$ sums to 1 for every movement phase $\lambda \in \Lambda$, $\mathbf{C}: \boldsymbol{\Lambda} \rightarrow \mathbb{R}^{N \times N}$, and $\mathbf{D}(\lambda) \in \mathbb{R}^{1 \times N}$. Eigenvalues of matrix $\mathbf{C}(\lambda)$ are all placed inside a disk of radius $r_{\lambda}<0$ with center at the origin. Note that the $i$-th entry of matrix $\mathbf{D}: \boldsymbol{\Lambda} \rightarrow \mathbb{R}^{1 \times N}$ specifies the fraction of traffic flow exiting the NOIR from node $i \in \mathcal{V}_{R}$. Traffic dynamics at non-exit nodes is given by

$$
\mathbf{x}_{R}(k+1)=\mathbf{C}(\lambda) \mathbf{x}_{R}(k)+\mathbf{g}_{R}(k),
$$

where $\mathbf{x}_{R}(k)=\left[\begin{array}{lll}\rho_{1}(k) & \cdots & \rho_{N}(k)\end{array}\right]^{T}$.

\section{PRoblem SPECificAtion}

Linear Temporal Logic (LTL) is used to specify the conservation-based traffic coordination dynamics [37] and present the feasibility conditions. Every LTL formula consists of a set of atomic propositions, logical operators, and temporal operators. Logical operators include $\neg$ ("negation"), $\vee$ ("disjunction"), $\wedge$ ("conjunction"), and $\Rightarrow$ ("implication").
LTL formulae also use temporal operators $\square$ ("always"), $\bigcirc$ ("next"), $\diamond$ ("eventually"), and $\mathcal{U}$ ("until").

We extend discrete-time LTL with the syntactic sugar $\square\left\{0, \ldots, N_{\tau}\right\}$ to specify satisfaction of a certain property in the next $N_{\tau}+1$ time steps. More specifically, $\square_{\left\{0, \ldots, N_{\tau}\right\}} \varphi$ at discrete time $k$ if and only if $\varphi$ is satisfied at discrete times $k$ to time $k+N_{\tau}$ [36].

The problem of traffic coordination can be formally specified by a finite-state abstraction defined by tuple

$$
\mathrm{M}=(\mathcal{S}, \mathcal{A}, \mathcal{H}, \mathrm{C}),
$$

where $\mathcal{S}$ is the state set, $\mathcal{A}$ is the discrete action set, $\mathcal{H}$ : $\mathcal{S} \times \mathcal{A} \rightarrow \mathcal{S}$ is the state transition relation, and $\mathrm{C}: \mathcal{S} \times \mathcal{A} \rightarrow \mathbb{R}_{+}$ is the immediate cost function.

\section{A. State set $\mathcal{S}$}

Set $\mathcal{S}$ is mathematically defined by

$$
\mathcal{S}=\left\{s=(\mathbf{x}, \mathbf{g}, \lambda, \tau) \mid \mathbf{x} \in \mathbf{X}, \mathbf{g} \in \mathbf{G}, \lambda \in \boldsymbol{\Lambda}, \tau \in\{0,1\}^{m}\right\},
$$

where the traffic density vector $\mathbf{x}=\left[\begin{array}{lll}\rho_{1} & \cdots & \rho_{N}\end{array}\right]^{T} \in \mathbb{R}^{N+1}$ and input vector $\mathbf{g} \in \mathbf{G} \in \mathbb{R}^{N_{\text {in }} \times 1}$ were introduced in Section III and $\mathbf{X}$ and $\mathbf{G}$ are compact sets. Also, $\lambda=\left(\lambda_{1, \zeta_{1}}, \cdots, \lambda_{m, \zeta_{m}}\right) \in \boldsymbol{\Lambda}$ is a movement phase, and $\tau=\left(\tau_{1}, \cdots, \tau_{m}\right) \in\{0,1\}^{m}$ where $\tau_{i} \in$ $\{0,1\}$ is the activation index at junction $i \in \mathcal{W}$. An execution of the proposed system is expressed by $s=s_{0} s_{1} s_{2}, \cdots$ where $s_{k}=\left(\mathbf{x}[k], \mathbf{g}_{k}, \lambda[k], \tau[k]\right)$ is the state of the system at time $k$.

Feasibility Condition 1: Traffic density, defined as the number of cars at a road element, is a positive quantity everywhere in the NOIR. It is also assumed that every road element has maximum capacity $\rho_{\max }$. Therefore, the number of cars cannot exceed $\rho_{\max }$ in any road element $i \in \mathcal{V}$. These two requirements can be formally specified as follows:

$$
\bigwedge_{i \in \mathcal{V}} \square_{\left\{0, \ldots, N_{\tau}\right\}}\left(\rho_{i} \geq 0 \wedge \rho_{i} \leq \rho_{\max }\right)
$$

If feasibility condition $\Phi_{1}$ is satisfied at every road element, then traffic over-saturation is avoided everywhere in the NOIR, at every discrete time $k$.

Optional Condition 2: Boundary inflow should satisfy the following feasibility condition at every discrete time $k$ :

$$
\square_{\left\{0, \ldots, N_{\tau}\right\}}\left(\sum_{i \in \mathcal{V}_{i n}} u_{i}=u_{0}\right) .
$$

Boundary condition $\Phi_{2}$ constrains the number of vehicles entering the NOIR to be exactly $u_{0}$ at any time $k$. Note that $u_{0}$ is an upper bound on vehicles entering the NOIR. However, in the simulation results presented, traffic demand is significant such that the NOIR is maximally utilized by as many vehicles as possible. 


\section{B. Action Set $\mathcal{A}$}

Action set $\mathcal{A}: \boldsymbol{\Lambda} \times \mathcal{T} \rightarrow \boldsymbol{\Lambda}$ assigns the next acceptable movement at every junction $i \in \mathcal{W}$, given the current NOIR activation index $\tau \in \mathcal{T}=\{0,1\}^{m}$ and movement phase $\lambda=$ $\left(\lambda_{1}, \cdots, \lambda_{m}\right)$, i.e. $\tau=\left(\tau_{1}, \cdots, \tau_{m}\right), \tau_{i} \in\{0,1\}, i \in \mathcal{W}$. We write $\lambda_{i}^{+}$for the value of $\lambda_{i}$ in the next state, i.e. $\lambda_{i}^{+}(k)=\lambda_{i}(k+1)$, and similarly for other variables. Actions are constrained and must satisfy one of the following LTL formula:

$$
\begin{gathered}
\left(\tau_{i}=0\right) \Rightarrow\left(\left(\lambda_{i}, \lambda_{i}^{+}\right) \in \Xi_{i} \vee\left(\lambda_{i}^{+}=\lambda_{i}\right)\right), \quad\left(\Phi_{3, i}\right) \\
\left(\tau_{i}=1\right) \Rightarrow\left(\lambda_{i}, \lambda_{i}^{+}\right) \in \Xi_{i}, \quad\left(\Phi_{4, i}\right)
\end{gathered}
$$

Combining $\left(\overline{\Phi_{3, i}}\right)$ and $\left(\overline{\Phi_{4, i}}\right)$, the next movement phase must satisfy the following LTL formula:

$$
\left.\bigwedge_{i \in \mathcal{W}} \square_{\left\{0, \ldots, N_{\tau}\right\}}\left(\left(\left(\lambda_{i}, \lambda_{i}^{+}\right) \in \Xi_{i}\right) \mathcal{U}\left(\tau_{i}=1\right)\right) \vee\left(\left(\lambda_{i}, \lambda_{i}^{+}\right) \in \Xi_{i}\right)\right) .
$$

Remark 1. Set $\mathcal{A}(\lambda, \tau) \subset \boldsymbol{\Lambda}$ is defined as follows:

$\mathcal{A}(\lambda, \tau)=\left\{\lambda^{+} \in \boldsymbol{\Lambda} \mid \forall i \in \mathcal{W},\left(\lambda_{i}, \lambda_{i}^{+}\right) \in \Xi_{i} \vee\left(\tau_{i}=0 \wedge \lambda_{i}^{+}=\lambda_{i}\right)\right\}$.

\section{State Transition Function}

The state transition relation $\mathcal{H}$ defines transition from "current" state $s=(\mathbf{x}, \mathbf{g}, \lambda, \tau) \in \mathcal{S}$ to "next" state $s^{+}=$ $\left(\mathbf{x}^{+}, \mathbf{g}^{+}, \lambda^{+}, \tau^{+}\right) \in \mathcal{S}$ given action $a(\lambda, \tau) \in \mathcal{A}(\lambda, \tau)$. Current and next movement phases must satisfy condition $\left(\Phi_{6}\right)$ below.

Transition of current activation index $\tau$ must satisfy the following properties:

$$
\bigwedge_{i \in \mathcal{W}}\left(\left(\tau_{i}^{+}=0\right) \mathcal{U}\left(T_{i}=T_{L, i}\right)\right) .
$$

Note that the $T_{i}$ is reset every time movement phase is updated at junction $i \in \mathcal{W}$. This requirement is formally specified as follows:

$$
\forall i \in \mathcal{W}, \quad\left(\lambda_{i}^{+} \neq \lambda_{i}\right) \Rightarrow\left(T_{i}^{+}=0\right)
$$

This paper assumes that $g_{i}=d_{i}$ is a Gaussian process for $i \in \mathcal{V}_{I}$ is an non-inlet road, i.e. $d_{i} \sim \mathcal{N}\left(\bar{d}_{i}, \sigma_{i}\right)$. Per Eq. (16), $g_{i}=$ $u_{i}$ for $i \in \mathcal{V}_{i n}$ where $u_{i}$ is determined as the solution of a receding horizon optimization problem presented in Section V] Therefore

$$
\left(\bigwedge_{i \in \mathcal{V}_{i n}} g_{i}^{+}=u_{i}^{+}\right) \wedge\left(\bigwedge_{i \in \mathcal{V}_{I}} g_{i}^{+}=y_{i}\right) \wedge\left(\bigwedge_{i \in \mathcal{V}_{E}} g_{i}^{+}=0\right) .
$$

Transition of $\mathbf{x}$ is governed by [15], thus

$$
\mathbf{x}^{+}=\mathbf{A}(\lambda) \mathbf{x}+\mathbf{g}
$$

where $\lambda \in \boldsymbol{\Lambda}$.

\section{Cost Function}

Given Eq. (15), an $N_{\tau}$-step expected transition is given by

$$
\mathbf{x}_{N_{\tau}+1}=\boldsymbol{\Theta}_{h}(\lambda) \mathbf{x}_{1}+\boldsymbol{\Gamma}_{N_{\tau}}\left[\begin{array}{c}
\mathbf{g}_{1} \\
\vdots \\
\mathbf{g}_{N_{\tau}}
\end{array}\right],
$$

where $\mathbf{g}_{1}, \cdots, \mathbf{g}_{N_{\tau}} \in \mathbf{G}, \mathbf{x}_{1} \in \mathbf{X}, \lambda \in \mathbf{\Lambda}$,

$$
\boldsymbol{\Theta}_{N_{\tau}}(\lambda)=\mathbf{A}^{N_{\tau}}(\lambda)
$$

and

$$
\boldsymbol{\Gamma}_{N_{\tau}}=\left[\begin{array}{llll}
\boldsymbol{\Theta}_{N_{\tau}-1} & \cdots & \boldsymbol{\Theta}_{1} & \mathbf{I}
\end{array}\right] \in \mathbb{R}^{(N+1) \times N_{\tau}(N+1)} .
$$

The cost function $\mathrm{C}$ is defined by

$$
\begin{aligned}
\mathrm{C}\left(\mathbf{x}, \mathbf{g}_{1}, \cdots, \mathbf{g}_{N_{\tau}}, \lambda\right) & =\sum_{h=1}^{N_{\tau}} \mathbf{x}_{h+1}^{T} \mathbf{F}^{T} \mathbf{F} \mathbf{x}_{h+1} \\
& =\left[\begin{array}{llll}
\mathbf{x}_{1}^{T} & \mathbf{g}_{1}^{T} & \cdots & \mathbf{g}_{N_{\tau}}^{T}
\end{array}\right] \mathbf{W}\left[\begin{array}{c}
\mathbf{x}_{1} \\
\mathbf{g}_{1} \\
\vdots \\
\mathbf{g}_{N_{\tau}}
\end{array}\right]
\end{aligned}
$$

where

$$
\mathbf{F}=\left[\begin{array}{cc}
\mathbf{I}_{N} & \mathbf{0}_{N \times 1} \\
\mathbf{0}_{1 \times N} & 0
\end{array}\right],
$$

and

$$
\mathbf{W}=\left[\begin{array}{cc}
\sum_{h=1}^{N_{\tau}} \boldsymbol{\Theta}_{h}^{T} \mathbf{F}^{T} \mathbf{F} \Theta_{h} & \sum_{h=1}^{N_{\tau}-1} \mathbf{\Theta}_{N_{\tau}}^{T} \mathbf{F}^{T} \mathbf{F} \boldsymbol{\Gamma}_{h} \\
\sum_{h=1}^{N_{\tau}} \mathbf{\Gamma}_{h}^{T} \mathbf{F}^{T} \mathbf{F} \Theta_{N_{\tau}} & \sum_{h=1}^{N_{\tau}} \mathbf{\Gamma}_{h}^{T} \mathbf{F}^{T} \mathbf{F} \boldsymbol{\Gamma}_{h}
\end{array}\right] .
$$

\section{TRAFic Congestion CONTROL}

The objective of the traffic congestion control is to determine optimal inflow and movement phase such that cost function $\mathrm{C}$, defined in Eq. (25), is minimized. Optimal traffic inflow is assigned with MPC while optimal movement phases are assigned as the solution of a $\mathrm{RHO}$ problem.

The optimal boundary inflow $\mathbf{g}_{1}^{*}$ is assigned by solving the following optimization problem:

$\mathbf{x} \in \mathbf{X}, \lambda \in \mathbf{\Lambda}, \quad\left(\mathbf{g}_{1}^{*}, \cdots, \mathbf{g}_{N_{\tau}}^{*}\right)=\underset{\mathbf{g}_{1}, \cdots, \mathbf{g}_{N_{\tau}} \in \mathbf{G}}{\operatorname{argmin}} \mathrm{C}\left(\mathbf{x}, \mathbf{g}_{1}, \cdots, \mathbf{g}_{N_{\tau}}, \lambda\right)$,

subject to the conditions $\Phi_{1}$ and $\Phi_{2}$.

The optimal movement phase $\lambda^{+*}$ is assigned by solving the following optimization problem:

$$
\begin{aligned}
& \mathbf{x} \in \mathbf{X}, \mathbf{g}_{1}, \cdots, \mathbf{g}_{N_{\tau}} \in \mathbf{G}, \lambda \in \mathbf{\Lambda}, \\
& \lambda^{+*}=\underset{\lambda^{+} \in \mathcal{H}(\lambda, \tau)}{\operatorname{argmin}} \mathrm{C}\left(\mathbf{x}, \mathbf{g}_{1}, \cdots, \mathbf{g}_{N_{\tau}}, \lambda\right),
\end{aligned}
$$

subject to the following conditions $\bigwedge_{i \in \mathcal{W}} \Phi_{i, 3}, \bigwedge_{i \in \mathcal{W}} \Phi_{i, 4}$, and $\Phi_{5}$. 


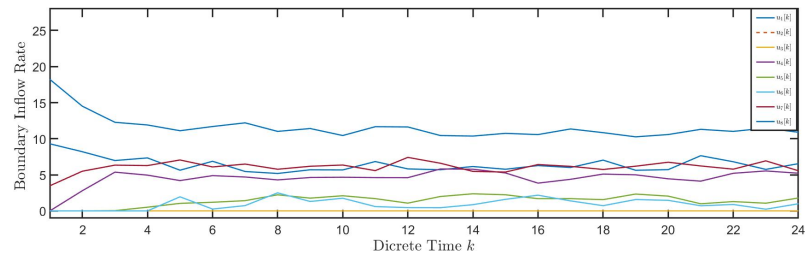

Fig. 2: Optimal boundary inflow rates $u_{1}$ through $u_{8}$ versus discrete time $k$.

\section{VI.Simulation RESULTS}

Traffic coordination is investigated in simulation for the example NOIR shown in Fig. 11 (a) consisting of $N=53$ unidirectional roads. Traffic coordination is controlled through the NOIR inlet boundary nodes defined by $\mathcal{V}_{i n}=\{1, \cdots, 8\}$ and traffic signals at junction nodes $\mathcal{W}=\{1, \cdots, 17\}$.

This paper assumes that the time interval between two consecutive discrete times $k$ and $k+1$ is $\Delta t=30 \mathrm{~s}$. It is assumed that the inflow $y_{i}=\frac{1}{2} \pm 0.5$ is randomly entered through every road element $i \in \mathcal{V}_{I}$. For simulation $u_{0}=31$ is chosen. Therefore, a total of 31 vehicles are allowed to enter the NOIR through the NOIR inlet boundary road elements at every discrete time $k$. Traffic coordination is controlled through the ramp meter at the NOIR boundary road elements and traffic signals at NOIR intersections by solving the optimization problem developed in Section $\mathrm{V}$.

In Fig. 2, boundary inflow rates $u_{1}$ through $u_{8}$ are plotted versus time for $k=1, \cdots, 100$. For the simulation, $\rho_{\max }=40$ is considered. Fig. 4 plots traffic density $\rho_{i}$ at every road element $i \in \mathcal{V}$ versus discrete time $k$. It is seen that $\left.\rho_{(} k\right)<\rho_{\max }=40$ at every discrete time $k$. Thus, traffic oversaturation is ensured. Also, the total traffic density $r_{\text {net }}(k)=\mathbf{1}_{1 \times N} \mathbf{x}_{R}(k)$ is plotted versus discrete time $k$ in Fig. 5. For simulation, we choose $T_{L, i}=3$. Therefore, a movement phase cannot be active more than $3 \times \Delta T=90 \mathrm{~s}$. A movement phase at junction $i \in \mathcal{W}$ is represented by a directed tree containing a root node and terminal nodes per the example movement phase shown in Fig. 1 (b). The root node represents the active road with incoming traffic flow, and terminal nodes represent the active outgoing roads. In Fig. 3, active incoming roads are shown at NOIR junctions $1, \cdots, 13 \in \mathcal{W}$ for $k=1, \cdots, 24$.

Fig. 5 plots the net traffic density of the NOIR versus discrete time $k$ for $k=1, \cdots, 24$. It is seen that net traffic density reaches the steady-state value in about eight time steps while traffic consistently enters and leaves the NOIR.

\section{CONCLUSION}

This paper offers a physics-inspired approach to model and control traffic coordination in a network of interconnected roads (NOIR). Traffic coordination modeled as a Markov process is obtained by spatial and temporal discretization of the mass conservation continuity equation. We showed how traffic congestion can be effectively controlled through ramp meters and traffic signals located at boundaries and junctions of the NOIR. In particular, MPC is applied to control the boundary

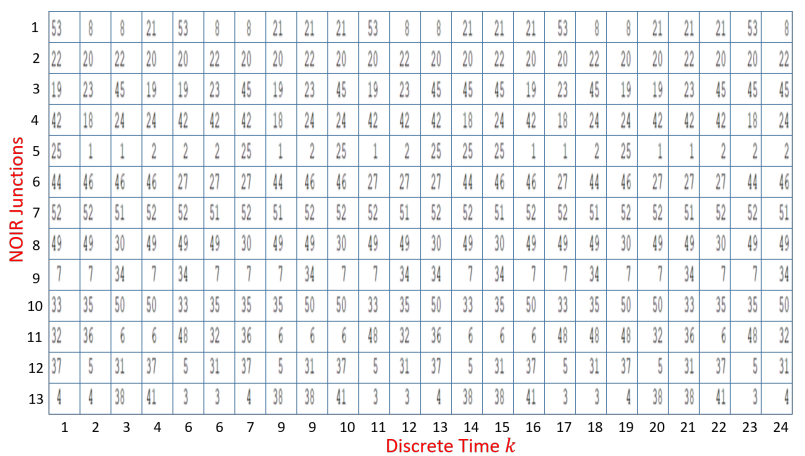

Fig. 3: Optimal movement phases at NOIR junctions at $k=$ $1, \cdots, 24$.

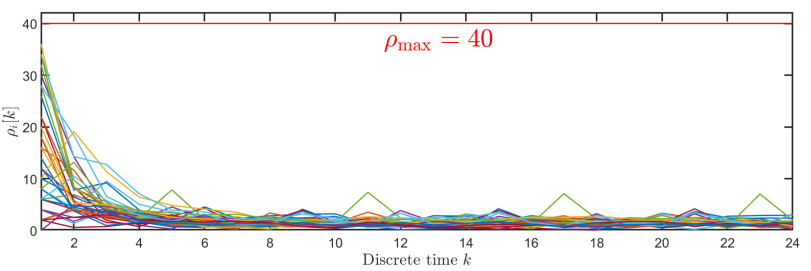

Fig. 4: Traffic density at every NOIR road for $k=1, \cdots, 24$.

inflow while a RHO planner optimizes movement phases commanded by traffic signals at NOIR junctions. Simulation results show that integration of boundary and signal controls can effectively manage urban traffic congestion.

\section{VIII.ACKNOWLEDGEMENT}

This work has been supported by the National Science Foundation under Award Nos. 1914581 and 1739525. The authors gratefully thank Professor Ella Atkins for the useful comments on this paper.

\section{REFERENCES}

[1] C. F. Daganzo, "The cell transmission model, part ii: network traffic," Transport. Research Part B: Methodological, vol. 29, no. 2, pp. 79-93, 1995.

[2] G. Gomes and R. Horowitz, "Optimal freeway ramp metering using the asymmetric cell transmission model," Transportation Research Part C: Emerging Technologies, vol. 14, no. 4, pp. 244-262, 2006.

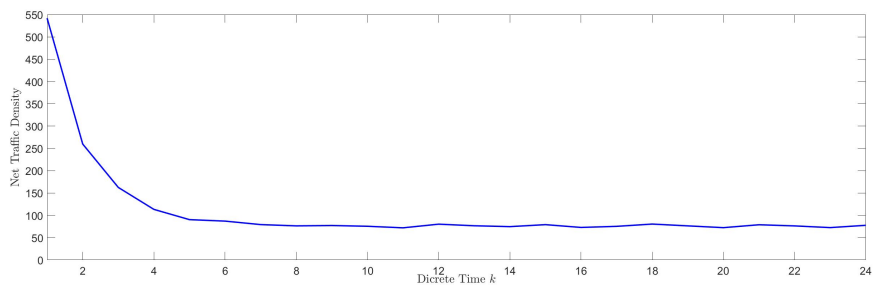

Fig. 5: Total traffic density of the NOIR vs. discrete time $k$. 
[3] D. I. Robertson, "Transyt: a traffic network study tool," Road Research Laboratory /UK, 1969.

[4] G. Tiwari, J. Fazio, S. Gaurav, and N. Chatteerjee, "Continuity equation validation for nonhomogeneous traffic," Journal of Transportation Engineering, vol. 134, no. 3, pp. 118-127, 2008.

[5] P. Balaji and D. Srinivasan, "Type-2 fuzzy logic based urban traffic management," Engineering Applications of Artificial Intelligence, vol. 24, no. 1, pp. 12-22, 2011.

[6] S. Chiu, "Adaptive traffic signal control using fuzzy logic," in Proceedings of the Intelligent Vehicles92 Symposium. IEEE, 1992, pp. 98-107.

[7] J. Zhang, W. Klingsch, A. Schadschneider, and A. Seyfried, "Ordering in bidirectional pedestrian flows and its influence on the fundamental diagram," Journal of Statistical Mechanics: Theory and Experiment, vol. 2012, no. 02, p. P02002, 2012.

[8] —, "Transitions in pedestrian fundamental diagrams of straight corridors and t-junctions," Journal of Statistical Mechanics: Theory and Experiment, vol. 2011, no. 06, p. P06004, 2011.

[9] K. Han, B. Piccoli, T. L. Friesz, and T. Yao, "A continuous-time link-based kinematic wave model for dynamic traffic networks," arXiv preprint arXiv:1208.5141, vol. 4, p. 35, 2012.

[10] G. Gentile, L. Meschini, and N. Papola, "Spillback congestion in dynamic traffic assignment: a macroscopic flow model with timevarying bottlenecks," Transportation Research Part B: Methodological, vol. 41, no. 10 , pp. 1114-1138, 2007

[11] V. Adamo, V. Astarita, M. Florian, M. Mahut, and J. Wu, "Modelling the spill-back of congestion in link based dynamic network loading models: a simulation model with application," in 14th International Symposium on Transportation and Traffic Theory, 1999

[12] G. Gentile, "Using the general link transmission model in a dynamic traffic assignment to simulate congestion on urban networks," Transportation Research Procedia, vol. 5, pp. 66-81, 2015.

[13] J. Long, Z. Gao, H. Ren, and A. Lian, "Urban traffic congestion propagation and bottleneck identification," Science in China Series F: Information Sciences, vol. 51, no. 7, p. 948, 2008.

[14] S. Jafari and K. Savla, "On structural properties of feedback optimal control of traffic flow under the cell transmission model," arXiv preprint arXiv:1805.11271, 2018

[15] S. Peeta and A. K. Ziliaskopoulos, "Foundations of dynamic traffic assignment: The past, the present and the future," Networks and spatial economics, vol. 1, no. 3-4, pp. 233-265, 2001.

[16] B. N. Janson, "Dynamic traffic assignment for urban road networks," Transportation Research Part B: Methodological, vol. 25, no. 2-3, pp. 143-161, 1991.

[17] C. F. Daganzo, "The cell transmission model: A dynamic representation of highway traffic consistent with the hydrodynamic theory," Transportation Research Part B: Methodological, vol. 28, no. 4, pp. 269-287, 1994

[18] S. Lin, B. De Schutter, Y. Xi, and H. Hellendoorn, "Efficient networkwide model-based predictive control for urban traffic networks," Transportation Research Part C: Emerging Technologies, vol. 24, pp. 122 $140,2012$.

[19] A. Jamshidnejad, I. Papamichail, M. Papageorgiou, and B. De Schutter, "Sustainable model-predictive control in urban traffic networks: Efficient solution based on general smoothening methods," IEEE Transactions on Control Systems Technology, vol. 26, no. 3, pp. 813-827, 2018.

[20] T. Tettamanti, T. Luspay, B. Kulcsar, T. Péni, and I. Varga, "Robust control for urban road traffic networks," IEEE Transactions on Intelligent Transportation Systems, vol. 15, no. 1, pp. 385-398, 2014.

[21] L. D. Baskar, B. De Schutter, and H. Hellendoorn, "Traffic management for automated highway systems using model-based predictive control,' IEEE Transactions on Intelligent Transportation Systems, vol. 13, no. 2 , pp. 838-847, 2012.

[22] H. M. Kammoun, I. Kallel, J. Casillas, A. Abraham, and A. M. Alimi, "Adapt-traf: An adaptive multiagent road traffic management system based on hybrid ant-hierarchical fuzzy model," Transportation Research Part C: Emerging Technologies, vol. 42, pp. 147-167, 2014.

[23] M. Collotta, L. L. Bello, and G. Pau, "A novel approach for dynamic traffic lights management based on wireless sensor networks and multiple fuzzy logic controllers," Expert Systems with Applications, vol. 42 , no. 13 , pp. 5403-5415, 2015

[24] G. Pau, T. Campisi, A. Canale, A. Severino, M. Collotta, and G. Tesoriere, "Smart pedestrian crossing management at traffic light junctions through a fuzzy-based approach," Future Internet, vol. 10, no. 2, p. 15,2018

[25] N. Yusupbekov, A. Marakhimov, H. Igamberdiev, and S. X. Umarov, "An adaptive fuzzy-logic traffic control system in conditions of saturated transport stream," The Scientific World Journal, vol. 2016, 2016.

[26] F. Moretti, S. Pizzuti, S. Panzieri, and M. Annunziato, "Urban traffic flow forecasting through statistical and neural network bagging ensemble hybrid modeling," Neurocomputing, vol. 167, pp. 3-7, 2015

[27] J. Tang, F. Liu, Y. Zou, W. Zhang, and Y. Wang, "An improved fuzzy neural network for traffic speed prediction considering periodic characteristic," IEEE Transactions on Intelligent Transportation Systems, vol. 18, no. 9, pp. 2340-2350, 2017.

[28] S. Akhter, R. Rahman, and A. Islam, "Neural network (nn) based route weight computation for bi-directional traffic management system," International Journal of Applied Evolutionary Computation (IJAEC), vol. 7, no. 4, pp. 45-59, 2016.

[29] K. Kumar, M. Parida, and V. K. Katiyar, "Short term traffic flow prediction in heterogeneous condition using artificial neural network," Transport, vol. 30, no. 4, pp. 397-405, 2015.

[30] H. Y. Ong and M. J. Kochenderfer, "Markov decision process-based distributed conflict resolution for drone air traffic management," Journal of Guidance, Control, and Dynamics, pp. 69-80, 2016.

[31] R. Haijema and J. van der Wal, "An mdp decomposition approach for traffic control at isolated signalized intersections," Probability in the Engineering and Info. Sciences, vol. 22, no. 4, pp. 587-602, 2008.

[32] S. Coogan, M. Arcak, and C. Belta, "Formal methods for control of traffic flow: Automated control synthesis from finite-state transition models," IEEE Control Systems Magazine, vol. 37, no. 2, pp. 109-128, 2017.

[33] S. Coogan, E. A. Gol, M. Arcak, and C. Belta, "Traffic network control from temporal logic specifications," IEEE Transactions on Control of Network Systems, vol. 3, no. 2, pp. 162-172, 2015.

[34] E. Christofa, I. Papamichail, and A. Skabardonis, "Person-based traffic responsive signal control optimization," IEEE Transactions on Intelligent Transportation Systems, vol. 14, no. 3, pp. 1278-1289, 2013.

[35] Y. Wang, W. Szeto, K. Han, and T. L. Friesz, "Dynamic traffic assignment: A review of the methodological advances for environmentally sustainable road transportation applications," Transportation Research Part B: Methodological, vol. 111, pp. 370-394, 2018.

[36] H. Rastgoftar, J.-B. Jeannin, and E. Atkins, An Integrative Behavioralbased Physics-Inspired Approach to Traffic Congestion Control, 2020, DynamicsSystemsControlConference

[37] T. Wongpiromsarn, U. Topcu, and R. M. Murray, "Receding horizon temporal logic planning for dynamical systems," in Proceedings of the 48h IEEE Conference on Decision and Control (CDC) held jointly with 2009 28th Chinese Control Conference. IEEE, 2009, pp. 5997-6004. 